\title{
The development of a novel test method to assess the durability of asphalt road-pavement materials
}

\author{
Bamber R K Blackman, Shuang Cui, Anthony J Kinloch, Ambrose C Taylor \\ Department of Mechanical Engineering, Imperial College London, South Kensington \\ Campus, London, SW7 2AZ, UK
}

Corresponding author: Dr. S. Cui

(s.cui@imperial.ac.uk, Tel: +44 2075895111 Ext 57093; Fax: +44 207594 7017)

\begin{abstract}
A better understanding of the adhesion mechanisms between the bitumen binder and the aggregate substrates, as employed in asphalt road pavements, is necessary to improve the durability of road-pavement materials. A new test protocol is outlined in the present paper to measure the characteristic fracture resistance of such bitumen-aggregate joints and to better understand the causes of failure of asphalt road surfaces, especially when associated with moisture ingress. The new test protocol is based upon a novel peel test which has been developed using a fracture mechanics approach to determine the adhesive fracture energy of bitumen-aggregate joints tested in both an unconditioned state (i.e. in the 'dry' state) and after being conditioned in an aqueous environment. Thus, the initial fracture resistance of the bitumen-aggregate can be assessed and the effects of any moisture-induced damage can be successfully quantified by the determination of the adhesive fracture energy. With these newly-developed techniques, the effects of using different aggregates and different bitumen grades may be quantitatively studied, since this test technique and recommended protocol may be readily adapted according to the requirements of different grades of bitumen, types of aggregate and required test conditions.
\end{abstract}

Keywords: C. Peel; D. Durability; D. Fracture; Asphalt 


\section{Introduction}

The road network is one of the most important elements of a modern transportation system. Across the United Kingdom, the total budget spent on road maintenance during $2009 / 10$ was of the order of $£ 3.8$ billion [1,2]. Thus, the significant costs and the disruption to traffic flows when maintaining the road network lead to the requirement for increasingly more durable road-pavement materials. The majority of road networks throughout the world are surfaced with asphalt mixtures. Asphalt is a composite material, consisting of mineral aggregates with bitumen as a binder. Bitumen is the sticky, black and highly viscous liquid, or semi-solid, present in crude oil and in some natural deposits; it is a substance classed as a pitch. It is composed primarily of a mixture of highly-condensed polycyclic aromatic hydrocarbons [3].

A major cause of failure of the road surface is due to moisture ingress and subsequent attack on the asphalt road-pavement material [4-6]. Moisture-damage is a particularly complicated mode of failure that may lead to the loss of stiffness and structural strength of the asphalt aggregate-bitumen mixture. This damage may arise from debonding and cracking at the binder-aggregate interface and/or a decrease in the mechanical properties of the binder, or occasionally even failure of the mineral aggregate [4-6]. Previous studies have indicated that the susceptibility of asphalt mixtures to moisture attack is related to the bitumen chemistry, aggregate mineralogy, surface texture of the aggregate and the adhesion between the bitumen binder and the aggregates [3,6-9]. In addition, the ambient conditions (including temperature, freeze-thaw cycles and wetting-drying cycles) can also affect significantly the durability of an asphalt road-pavement material $[10,11]$.

Numerous laboratory test methods have been developed to identify the durability of asphalt road-pavement materials and their response to moisture ingress [6,12-17]. These methods can be divided into two groups: (a) qualitative tests conducted on loose bitumencoated aggregate, such as the boiling test [13], and (b) quantitative tests conducted on compacted asphalt mixtures [14], such as the wheel-tracking test [15] and the Saturation Ageing Tensile Stiffness (SATS) test $[16,17]$. The relevant test specimens are typically conditioned in water to simulate in-service conditions and an assessment of any moistureinduced damage is made by dividing the conditioned modulus or strength by the corresponding unconditioned property, for example as in the freeze-thaw AASHTO T28399 procedure [18]. Although these approaches are realistic and logical in terms of 
simulating the in-service asphalt road-pavement materials, they are frequently too complex and too insensitive to allow differentiation between different types of bitumen binders and aggregates, and the nature of the bitumen-aggregate interface. Indeed, any actual study of the adhesion acting across the bitumen-aggregate interface is rare in these methods. Most importantly, the above methods do not measure any fundamental or characteristic fracture properties. Hence, they do not provide any understanding of the performance of asphalt road-pavement materials when exposed to ingressing moisture and do not provide any definitive guidance for selecting asphalt mixtures with an improved performance.

In addition to these laboratory test methods, a number of computational approaches have been developed to simulate the in-service conditions experienced by the asphalt roadpavement materials, and hence to attempt to predict the durability and moisture resistance of such materials, e.g. [19-23]. However, the predictions from these computational methods have been found to be inaccurate when compared to in-service observations. This is undoubtedly due to the need to understand in detail the adhesion between the bitumen binder and the aggregates, and how such interactions between the bitumen binder and the aggregates are affected by the presence of moisture and other external factors.

Thus, for the development of both improved laboratory test methods and more accurate computational in-service prediction models, it is necessary to develop new test methods to ascertain any loss of (a) the adhesion between the bitumen and the aggregates [24, 25], and (b) the cohesive properties of the bitumen binders [26, 27] themselves in different environments. The present work aims to establish such a test method and to propose a test protocol to assess the degree of adhesion between the bitumen and the aggregates and/or the cohesion within the binder, as a function of the test environment. We will also seek to identify the degradation mechanisms operating when the asphalt road-pavement materials are subjected to the various aqueous environmental test conditions.

\section{Experimental Studies}

\subsection{Selection of the test method}

In the view of the deficiencies of the current test methods mentioned above, a more fundamental and direct measurement of both the adhesive and cohesive fracture properties of asphalt road-pavement materials is clearly needed. There are many test methods to be found in the scientific literature and international standards for measuring 
the fracture of interfaces and adhesive joints. Bitumen is a viscoelastic material [3] and hence the mechanical properties of bitumen are very sensitive to the ambient temperature and the test speed employed. Indeed, bitumen can display both glassy behaviour and viscous behaviour, depending on the combination of the test temperature and strain-rate employed. At room temperature, most grades of bitumen are very viscous and undergo viscoelastic deformation during loading, which makes fracture test methods based on an assumption of overall linear-elastic deformation, such as the tapered double-cantilever beam (TDCB) test method [28], unsuitable to assess asphalt mixtures.

On the other hand, the elastic-plastic peel test is one of the most frequently used test methods for assessing the failure of flexible laminates [29-32], and has therefore been selected due to the viscoelastic and relatively low-modulus characteristics of the bitumen binders. Furthermore, the peel test can be readily conducted under both 'dry' and 'wet' conditions. The adhesive fracture energy, $G_{A}$, calculated from the measured peel force represents the crack resistance of the interface or the bitumen binder, depending on the locus of failure observed, and hence of the corresponding asphalt road-pavement material.

\subsection{Introduction to the materials}

The principal constituents of asphalt road-pavement materials are mineral aggregates and bitumen binders. The properties of the mineral aggregates have a significant influence on the behaviour of asphalt mixtures. Limestone is known to possess a relatively good moisture resistance [7] and was therefore selected for the present studies as the standard aggregate material. Limestone belongs to the group of sedimentary rocks and is largely composed of calcium carbonate $\left(\mathrm{CaCO}_{3}\right)$ [3]. Most limestone is formed by the deposition and consolidation of skeletal fragments of marine organisms, although a few originate via chemical precipitation from lake or ocean water. Bitumen is the sticky, black and highly viscous liquid, or semi-solid, extracted from crude oil during refining. It is a colloidal system consisting of relatively high molecular-weight asphaltene micelles dispersed in a lower molecular-weight medium [3]. The principal constituents of bitumen are carbon $(>80 \%)$ and hydrogen $(\sim 10 \%)$, plus a small amount of other elements, such as nitrogen, oxygen, and sulphur [3]. It is composed primarily of a mixture of highly-condensed polycyclic aromatic hydrocarbons. However, the precise chemical composition of bitumen and hence its properties, depend on the crude oil source. There are a wide variety of different bitumens with different chemical compositions and hence physical properties, and they are classified by various specifications. The 'penetration grade' is a commonly used 
specification [3]. The 'penetration' number is defined as the distance (expressed in tenths of a millimetre) travelled by a needle into the bitumen under a known load, at a known temperature for a known time [3]. Penetration grades are usually referred to without stating the units and listed as a range of penetration (pen) values, such as '40/60 pen'. The lower the range of penetration values, the harder the bitumen. In this work, three bitumen binders provided by Shell Bitumen UK (Manchester) were used: 70/100 pen (soft), 40/60 pen (medium) and 10/20 pen (hard).

\subsection{Preparation of the peel test}

In a peel test, a flexible substrate and a rigid substrate are bonded using an adhesive, as illustrated in Figure 1. In this work, the aggregate formed the rigid substrate, which was bonded to the peel arm (i.e. a relatively flexible strip simply used to support the soft bitumen) using the various bitumen binders as the adhesive layer. The aggregate rigid substrates were wet-sawn from limestone boulders to a size of $200 \mathrm{~mm}$ long, $20 \mathrm{~mm}$ wide and $10 \mathrm{~mm}$ thick. The limestone used was $>98 \%$ calcite $\left(\mathrm{CaCO}_{3}\right)$, and the sawn surface has a similar roughness to that of the crushed aggregate used for asphalt. The rigid aggregate and the flexible peel arm were adhered along most of their length via the bitumen layer. However, to allow the peel arm to be gripped during testing, a length of unbonded interface, nominally $30 \mathrm{~mm}$ in length, was created by using a poly(tetrafluoroethylene) (PTFE) release film which was $13 \mu \mathrm{m}$ thick. The flexible peel arm must obviously have very good adhesion to the various bitumen binders, so that failure at the bitumen/peel-arm interface is avoided. Aluminium (Grade: EN AW-1200) of thickness $0.2 \mathrm{~mm}$ was found to be satisfactory to use for the peel-arm material, since it readily provided the necessary good adhesion to the binder.

The peel specimens were manufactured by the following steps, as detailed in Figure 2. Before bonding the joint, the surface of the aggregate was wiped gently using a damp paper towel to remove any dust. The aggregate was then placed in an oven at $50^{\circ} \mathrm{C}$ for 30 minutes to eliminate any surface moisture, except for the specimens with the moisture purposely introduced into the joint to study the effect of moisture damage, as described below. The bonding surface of the aluminium peel-arm was grit-blasted using 60-78 $\mu \mathrm{m}$ alumina particles at a pressure of 4 bar. To obtain a uniform treatment of the peel arm, a thick steel plate was used to support the peel arm during the treatment, since the peel arm was only $0.2 \mathrm{~mm}$ in thickness and was too soft to withstand the applied pressure. Most importantly, both sides of the peel arm were grit-blasted to eliminate the presence of any 
residual stresses which might arise from grit-blasting only one side of the peel arm. The peel arm was then rinsed with running water to remove any residual grit prior to being cleaned with acetone to remove any grease or oil.

The bitumen was preheated for 30 minutes at its application temperature (i.e. $150^{\circ} \mathrm{C}$ for the $40 / 60$ pen and $70 / 100$ pen bitumen and $180{ }^{\circ} \mathrm{C}$ for $10 / 20$ pen bitumen) prior to forming the joint, to enable it to be readily poured. These application temperatures are representative of production temperatures for asphalt using these various types of bitumen. The aluminium peel-arm was also preheated at $150^{\circ} \mathrm{C}$ for 5 minutes. As mentioned above, a $13 \mu \mathrm{m}$ thick release-film of PTFE, with dimensions of $30 \mathrm{~mm} \times 20 \mathrm{~mm}$, was placed on the bonding surface of the aggregate at one end, as shown in Figure 2 (a). The heated liquid bitumen was then poured evenly onto the aggregate surface, see Figure 2(b). The thickness of the adhesive layer was controlled via (a) a continuous, undulating, metal-wire spacer placed along the joint, and (b) two short metal-wire spacers inserted at either end of the joint, see Figure 2 (c). In the present work, $0.25 \mathrm{~mm}$ diameter copper wire was used for these spacers. An adhesive thickness, $h_{\mathrm{a}}$, of $0.25 \mathrm{~mm}$ was found to be satisfactory for the bitumen materials, as discussed below, and this thickness was adopted for all the present tests. The preheated aluminium peel-arm was then placed on the top of the bitumen layer, see Figure 2 (d), so that the peel arm overhung the starter film end of the aggregate. Gentle pressure was applied by placing a thick steel plate on top of the joint and clamping the whole joint to control the thickness of the bitumen layer. The pressure was uniformly distributed across the bonded area. The bonded specimen was cooled at ambient temperature overnight. Finally, the excess bitumen at the edges of the specimen was removed using a knife-blade, see Figure 2 (e). The bitumen-aggregate peel joints were always tested within a day of manufacture. Otherwise the viscoelastic nature of bitumen would lead to it exude out from the adhesive layer, reducing the thickness of the adhesive layer and hence introducing errors into the test results.

\subsection{Test procedure}

For each test, a minimum of three specimens were tested. The peel tests were conducted at controlled ambient conditions of $20 \pm 2^{\circ} \mathrm{C}$ and $50 \pm 5 \%$ relative humidity. The peel specimen was rigidly bolted to a frictionless sliding trolley on the crosshead of a universal testing machine, as shown in Figure 3. The free end of the flexible peel arm was bent to an applied peel angle of $90^{\circ}$ and connected to the load cell via a loading rod and grip. The peel test was conducted by setting the machine in motion at a constant speed of grip 
separation. In this way, the displacement of the movable end of the test machine was equivalent to the fracture length for a peel angle of $90^{\circ}$. A peel test speed of $10 \mathrm{~mm} / \mathrm{min}$ was used as a standard for a medium penetration grade of bitumen binder to ensure stable crack growth. However, as noted below, in some instances a small adjustment was made to the test speed to ensure that stable, as opposed to unstable, crack growth occurred during the peel test.

During the test, the peel force was recorded as a function of the displacement of the crosshead to initiate and propagate a peel fracture, as shown in Figure 4. A minimum length of $50 \mathrm{~mm}$ of peel fracture was always established. In Figure 4, an initiation region can be observed where the peel force rises steeply before the measured force settles to an approximately constant value during crack propagation. Once the steady-state crack propagation region was defined, the average value of the force over this region was calculated, and this was termed the steady-state peel propagation force, as shown in Figure 4. This steady-state propagation peel force was used to determine the values of the adhesive fracture energy, $G_{A}$. To acquire further information on the peel fracture behaviour, photographs of the side of the specimen were taken during the test.

In order to determine the value of the adhesive fracture energy, $G_{A}$, see below, it was necessary to calculate the plastic work dissipated in bending the peel arm, and any plastic energy dissipated in the peel arm in tension, during the test. Therefore, uniaxial tensile tests were conducted, at the same test speed as the peel test, using the aluminium which formed the peel arm material.

To understand the fracture mechanisms operating between the bitumen and the aggregate, it is important to study not only the crack resistance of the bitumen-aggregate joints but also to identify the locus of joint failure. In the present work the fracture surfaces were visually assessed after the peel test had been completed to determine the locus of failure.

\subsection{Data analysis}

The data analysis follows the procedures outlined in the ESIS TC4 protocol for the determination of the adhesive fracture energy for flexible laminates using peel tests [31]. The first step in the analysis is to determine the uncorrected adhesive fracture energy, $G$, [31, 32] via: 


$$
G=\frac{P}{b}(1-\cos \theta)
$$

where $P$ is the steady-state peel force, $b$ is the width of the specimen and $\theta$ is the applied peel angle, where $\theta=90^{\circ}$ in the present work. Now, Equation 1 assumes negligible tensile deformation in the peel arms, which is the case for the modest values of peel force attained in the present tests. However, it also assumes that negligible plastic deformation occurs in the peel arm during the peel test, which is clearly not the case. Thus, the adhesive fracture energy, $G_{A}$, is then obtained via:

$$
G_{A}=G-G_{\mathrm{P}}
$$

where $G_{p}$ is the energy associated with the plastic bending of the peel arm. This value is not negligible and constitutes a major component of the total energy dissipated. In order to determine the value of $G_{p}$, a tensile test is first performed on the peel arm material at the same equivalent test rate as the peel test. The value of $G_{p}$ is then determined using large displacement beam theory with modifications for plastic bending [31,32]. The data analysis was performed using the Microsoft Excel macro, 'IC Peel', which is freely available to download from the Imperial College website [33]. The full description of the calculation of $G_{p}$, and hence $G_{A}$, can be found in [32].

This analysis requires the tensile stress-strain curve for the peel arm to be measured and this is then an input parameter into the analysis. The stress-strain curve can be represented with either a bi-linear or a power-law fit to describe the initial elastic and then work hardening, plastic deformation of the peel arm. For the initial elastic deformation, when the strain in the peel arm is less than the yield strain, i.e. $\varepsilon \leq \varepsilon_{y}$ then the stress is:

$$
\sigma=E_{1} \varepsilon
$$

where $E_{1}$ is the elastic modulus of the peel arm. When the strain in the peel arm exceeds the elastic limit, i.e. when $\varepsilon>\varepsilon_{y}$ then according to the bi-linear model:

$$
\sigma=\sigma_{y}+\alpha E_{1}\left(\varepsilon-\varepsilon_{y}\right)
$$

where $\alpha$ is the ratio of the plastic to elastic modulus, $E_{2} / E_{1}$, and according to the powerlaw model:

$$
\sigma=\sigma_{y}\left(\frac{\varepsilon}{\varepsilon_{y}}\right)^{n}
$$

where $n$ is the work hardening coefficient for the peel arm material. 
Figures 5 (a) and (b) show a measured stress-strain curve for the aluminium peel arm used in the present work with the data fitted using (a) the bi-linear model and (b) the power law model. The ESIS TC4 protocol [31] permits either fitting technique to be used, although for the present tests the power-law model always gave the closer fit to the measured data and hence this one was used. Table 1 shows the effect on the value of $G_{p}$, and hence on $G_{A}$, of the fitting technique used. As can be seen in the Table, the difference between the two fitting techniques makes less than a $2.5 \%$ difference in the calculated values of $G_{p}$.

The recommended test report, as shown in Appendix 1, includes a description of the peel specimen, the tensile properties of the peel arm and the tensile modulus of the adhesive [31]. The test report shown in Appendix 1 is for a typical dry 40/60 pen bitumen-limestone joint with an aluminium peel arm. In addition, it is recommended that the test results should include a plot of the peel curve, i.e. the measured peel force versus displacement in the peel test, as shown in Figure 4. Further, the report should also include the tensile stress versus strain curve of the peel arm which is needed in order to calculate the plastic deformation energy associated with the peel arm, as shown in Figure 5 [31].

\subsection{The moisture conditioning of the bitumen-aggregate joints}

\subsubsection{Introduction}

Moisture-damage can reduce the structural strength of asphalt mixtures. This is due to the loss of the adhesion between the aggregate and the bitumen, and/or the loss of the cohesive strength within the bitumen binder. Characterisation of moisture-damage is a challenge since it involves various physical and mechanical processes. Therefore, it is important to ensure the consistency of test conditions, material properties and specimen dimensions, especially the bitumen thickness [34].

To study the response to moisture, water uptake tests using pre-dried (at $60^{\circ} \mathrm{C}$ for 24 hours) limestone and bulk bitumen specimens have been undertaken by submersing the specimens in water at $20^{\circ} \mathrm{C}$ for up to 10 days, during which time weight readings were recorded. From these tests, the calculated water diffusion coefficient for the limestone aggregate is $3.96 \times 10^{-9} \mathrm{~m}^{2} / \mathrm{s}$, which suggests that most water absorption for the limestone substrate took place within 10 hours. However, the water diffusion coefficient of bitumen is $1.96 \times 10^{-13} \mathrm{~m}^{2} / \mathrm{s}$, which implies bitumen binders do not take in high concentrations of water within the 10 days of immersion. Based on these results, the peel specimens were 
water-conditioned for periods ranging from 1 day to 10 days. The moisture was introduced into the peel specimen at two different stages in the specimen manufacturing processes, as illustrated in Figure 6, and as described below.

\subsubsection{Pre-bond conditioning}

Firstly, the aggregate was submersed in distilled water at $20^{\circ} \mathrm{C}$ before bonding the joint ('pre-bond' conditioning), as shown in Figure 6 (a). On removal from the water the conditioned aggregate was placed in an oven at $150^{\circ} \mathrm{C}$ for a short period (i.e. less than one minute) to remove the excess water on the bonding surface. (This practice eliminates the possibility of there being a layer of water on the surface which may prevent the bitumen binder contacting and fully wetting the aggregate when the joint is assembled.) The specimen was then bonded following the standard procedure described in Section 2.1, but without of course drying the aggregate in the oven for 30 minutes at $50^{\circ} \mathrm{C}$. By using this conditioning method, the effect of pre-existing moisture in the aggregate can be examined.

\subsubsection{Post-bond conditioning}

Secondly, moisture was introduced into the peel joint after bonding by submersing the completed specimen in distilled water at $20^{\circ} \mathrm{C}$ for various periods of time, as shown in Figure 6 (b). This procedure has been termed 'post-bond' moisture conditioning. In this conditioning method, water permeates into both the bitumen binder and the aggregate simultaneously, and may directly attack the interface. It is assumed that the aluminium peel arm is impermeable to water ingress. After removing the specimen from the water bath, the specimen was tested within a few hours. This method can more closely simulate the effect of moisture on the bitumen-aggregate interface, and therefore more closely simulates the in-service moisture attack on asphalt road surfaces.

\section{Results and Discussion}

\subsection{Peel force and fracture energy}

The specimens were made using a limestone aggregate (fixed-arm) and an aluminium peel arm bonded using 40/60 pen bitumen, which is a medium penetration grade binder and is widely used on roads. The tests were conducted at $20^{\circ} \mathrm{C}$ and at a speed of 10 $\mathrm{mm} / \mathrm{min}$, which was selected as the standard test speed for this medium penetration grade bitumen. 
Figure 7 shows both (a) satisfactory and (c) unsatisfactory peel test results, and the corresponding fracture surfaces in (b) and (d) respectively. After the initiation stage, the measured peel force remained at an approximately constant value, see Figure 7(a), indicating that the fracture progressed steadily (i.e. under steady-state conditions). This was consistent with the fracture surface, shown in Figure 7(b), in which a uniform layer of bitumen can be seen, indicating that cohesive failure occurred within the bitumen throughout the test. This peel test result was reproducible and was typical for a dry joint made using the 40/60 pen bitumen and limestone aggregate. An average peel force of 23 $\mathrm{N}$ was measured for the joints tested, from which the average adhesive fracture energy, $G_{A 0}$, of the dry specimen was calculated to be $633 \mathrm{~J} / \mathrm{m}^{2}$. A $\pm 9 \%$ variation in the value of the adhesive fracture energy was measured between specimens, which is reasonable as both aggregate and bitumen are natural materials, hence some variability is expected. This value of the adhesive fracture energy, $G_{A 0}=633 \mathrm{~J} / \mathrm{m}^{2}$, was used as the baseline in the study of the moisture susceptibility of this type of bitumen-aggregate joints.

In contrast, after the initiation stage in Figure 7(c), the peel force varied from $2 \mathrm{~N}$ to $10 \mathrm{~N}$, indicating unsteady (sometimes called 'stick-slip') growth of the crack. It is clearly seen in Figure $7(d)$ that the bitumen layer was thinner in some areas on the aggregate surface than in other areas, due to poor specimen preparation. The measured peel force, and thus the value of the adhesive fracture energy, $G_{A}$, is crucially dependent upon the thickness of the bitumen layer. In practice, the thinner the bitumen layer then the lower the value of the corresponding adhesive fracture energy. This is because there will be energy dissipation in the volume of a viscoelastic material, as discussed by Igarashi [35], and hence the adhesive fracture energy will depend on the adhesive layer thickness. A linear relationship between peel energy and adhesive layer thickness was observed by Igarashi [35] for rubbers and Giannis et al. [36] for sealants. This explains why the peel force in Figure 7(c) was, on average, lower than that of Figure 7(a). The peel test results shown in Figure 7(c) were therefore rejected, as the specimens were prepared with insufficient control of the bitumen thickness; new specimens were made with the correct $0.25 \mathrm{~mm}$ adhesive layer, and the results shown in Figure $7(a)$ and (b) were obtained.

\subsection{Effects of moisture}

3.2.1 Moisture conditioning of the bitumen-aggregate joints

To investigate the sensitivity of the adhesive fracture energy, $G_{A}$, to moisture, peel tests were undertaken using the moisture-conditioned bitumen-aggregate joints. These were 
pre-bond or post-bond conditioned in water, as described above, for between 1 and 10 days.

Figure 8 shows examples of both pre-bond and post-bond moisture-conditioned peel test results. These two specimens were made using the same materials and tested at the same conditions as the specimen in Figure 7(a), the only difference being that water had now been introduced into the specimens. It can be clearly seen that the measured peel force decreased for both types of moisture-conditioned specimens. This indicates that the bitumen-aggregate joints suffer a reduction in the value of the adhesive fracture energy with the presence of moisture compared to that of the dry joint. The images of the fracture surfaces give important additional information on the locus of failure. There was very little bitumen residue on the aggregate surface in Figure 8(b), signifying an interfacial failure (between the aggregate and the bitumen) in the pre-bond moisture-conditioned specimen. This suggests that the moisture present in the aggregate prior to forming the joint reduces the adhesion between the aggregate and the bitumen. In Figure 8(d), there is also very little bitumen residue on the aggregate surface, again signifying an interfacial fracture (between the aggregate and the bitumen) in the post-bond moisture-conditioned specimen.

The values of the adhesive fracture energy and the locus of failure for the peel joints are summarised in Table 2. The values in this Table are averaged from the results from at least three specimens at each immersion condition. The $G_{A}$ values for the pre-bond and post-bond conditioned specimens are significantly lower than those of the dry specimens. The value of the adhesive fracture energy also decreases with increasing conditioning time. These results can be used to quantify the extent of the loss of the joint strength due to moisture-damage for the bitumen-aggregate system: namely, the dimensionless ratio of the two fracture energies, $G_{A} / G_{A 0}$, represents the moisture sensitivity of the joints. When specimens were post-bond conditioned for 10 days, a $G_{A}$ value of $69 \mathrm{~J} / \mathrm{m}^{2}$ was calculated, which gives $G_{A} / G_{A 0}=0.11$. Hence, the joint strength after 10 days immersion in water is only $11 \%$ of that of the dry specimen, showing how fast and how significant the effect of moisture can be on the failure properties of the bitumen-aggregate joint.

The locus of failure gives important information on the change of fracture mechanism when moisture is introduced. Due to the good contrast between bitumen and most aggregates, the locus of failure can be obtained by direct visual observation of the fracture surfaces. For pre-bond conditioned specimens, the failure moved from the centre of the 
bitumen layer to the bitumen-aggregate interface, indicating that the presence of preexisting moisture in the aggregate can result in a loss of adhesion between the binder and the aggregates. For the post-bond conditioned specimens, interfacial failures were also observed, again implying that moisture can damage the substrate interface, which results in a decrease in the energy needed to rupture the bitumen-aggregate joint.

\subsubsection{Moisture conditioning of bitumen}

To differentiate the respective responses of the cohesive and adhesive properties of the bitumen-aggregate system to moisture-damage, an aluminium substrate was used to replace the aggregate. In this way, the effect of moisture-damage on the cohesive property of the bitumen binder alone can be studied since (a) the aluminium substrate does not absorb water, and (b) the bitumen-aluminium interface is not significantly attacked by ingressing moisture over the time period involved. Indeed, after post-bond conditioning of the bitumen-aluminium specimens, cohesive failure occurred, see Figure 9, and the summary given in Table 3 .

Several points of interest arise from the results shown in Table 3. Firstly, it may be seen that the measured adhesive fracture energy for the dry (i.e. unconditioned) bitumenaluminium joints is relatively high with a value of $633 \mathrm{~J} / \mathrm{m}^{2}$. This is an identical value to that of the dry (i.e. unconditioned) bitumen-limestone aggregate specimens, as may be seen from comparing the results given in Tables 1 and 3. Secondly, the calculated values of the adhesive fracture energy of the bitumen-aluminium joints were not significantly affected by the conditioning time, with $G_{A} / G_{A 0}=1$, within experimental variation, for all the bitumenaluminium specimens tested. Thirdly, the occurrence of cohesive failure during the peel tests for the bitumen-aluminium joints, and the independence of the corresponding values of the adhesive fracture energy, on the presence of moisture confirms that moistureinduced damage does not reduce significantly the cohesive properties of the bitumen binder. This is a sensible finding as bitumen has long been used as a waterproofing material [3].

\subsection{The soft and hard bitumen binders}

Due to the viscoelastic nature of the bitumen binders, a set of test parameters that work well for one bitumen binder may not work well for other bitumen binders of different penetration grades. In the present work it has been shown that, for the 40/60 pen bitumen binder, steady-state fracture occurs at a peel test speed of $10 \mathrm{~mm} / \mathrm{min}$ and at a test 
temperature of $20^{\circ} \mathrm{C}$. However, using the same test parameters, unsteady, stick-slip behaviour was observed in peel specimens formed using the hard 10/20 pen bitumen, as shown in Figure 10(a,b). Peel force versus displacement curves such as that shown in Figure 10(a) are generally not satisfactory for a reliable analysis, since no steady-state peel force can be obtained. Thus, no steady-state value for the adhesive fracture energy could be determined.

Modification of the test parameters was therefore investigated and has been shown to overcome this limitation, with steady-state peeling for other penetration grade binders being achieved. Based on the viscoelastic characteristics of bitumen, two approaches may be employed to eliminate the unsteady, stick-slip behaviour, i.e. by increasing the ambient temperature or by decreasing the test speed. As it is easier to adjust the test speed, this approach is preferred. Figure 10 (c,d) shows the peel curve of $10 / 20$ pen bitumen, and the corresponding fracture image, respectively, from tests conducted at a test speed of 2 $\mathrm{mm} / \mathrm{min}$, and at a test temperature of $20 \pm 2^{\circ} \mathrm{C}$ and $50 \pm 5 \%$ relative humidity. Steady-state fracture, with cohesive failure through the bitumen layer, is seen and this results in an approximately constant, steady-state peel force. This observation confirms that the unsteady, stick-slip behaviour of the harder bitumen grades can indeed be avoided by testing at a slower speed. Additionally, the standard test conditions for medium penetration grade bitumens may not work well for very soft bitumen binders. This problem may be overcome by increasing the test speed or by testing at a lower test temperature. However, for the 70/100 pen bitumen-aggregate joints used in the present study, a test speed of 10 $\mathrm{mm} / \mathrm{min}$ at $20 \pm 2{ }^{\circ} \mathrm{C}$ and $50 \pm 5 \%$ relative humidity (as used for the 40/60 pen bitumenaggregate joints) did give a steady-state peel fracture with cohesive failure through the bitumen layer.

As may be seen from Table 4 for the dry (i.e. unconditioned) bitumen-limestone specimens, the average values of the measured peel force and the adhesive fracture energy, $G_{A}$, of the $10 / 20$ pen bitumen joints were slightly higher than those of the $40 / 60$ pen bitumen joints. On the other hand, for the specimens using the 70/100 pen bitumen binder, the average value of the adhesive fracture energy was somewhat lower than that of the 40/60 pen bitumen joints. However, when the experimental variation of the results is considered, with a typical variation of $\pm 9 \%$, there is no significant difference between the values. 


\section{Conclusions}

It has been shown that the peel test is a suitable method to determine the adhesive fracture energy, $G_{A}$, of bitumen bonded to aggregate substrates, and this parameter provides a characteristic value for the failure of the joints. Further, that deducing the value of $G_{A}$ allows the quantification of the effects of moisture attack on the bitumen-aggregate joints, especially at the bitumen-aggregate interface. Thus, we can gain much valuable, quantitative, information on the performance of asphalt road-pavement materials.

For example, an adhesive fracture energy of $633 \mathrm{~J} / \mathrm{m}^{2}$ and cohesive failure through the bitumen binder were recorded for specimens made using a 40/60 pen bitumen binder and a limestone aggregate as the substrate and not exposed to aqueous conditions, i.e. tested 'dry'. By conditioning the peel joints at different stages of the manufacturing process for the specimens, the effects of moisture-induced damage on the fracture mechanism of bitumen-aggregate joint were then studied. When the specimens were subjected to a postbond condition, by immersing them in water for 10 days, only approximately $11 \%$ of the value of the initial adhesive fracture energy was retained. It has been found that the moisture-induced damage is mainly attributed to a reduction in the interfacial adhesion between the bitumen and the aggregate. The cohesive strength of the bitumen binder remains relatively unaffected by the presence of ingressing moisture.

Thus, the initial fracture resistance of the bitumen-aggregate can be assessed, and the effects of any moisture-induced damage can be successfully quantified, by the determination of the adhesive fracture energy. With these newly-developed techniques, the effects of using different aggregates and different bitumen grades may be quantitatively studied, since this test technique and recommended protocol may be readily adapted according to the requirements of different grades of bitumen, types of aggregate and required test conditions.

\section{Acknowledgements}

The authors would like to thank Prof. G.D. Airey and his group at the Nottingham Transportation Engineering Centre (NTEC) of the University of Nottingham for valuable discussions throughout this work, and for the supply of materials. The authors also gratefully acknowledge the financial support of the EPSRC (UK) under grant number EP/G039399/1. 


\section{References}

1. Annual Local Authority Road Maintenance (Alarm) Survey, Asphalt Industry Alliance, (2010). www.asphaltindustryalliance.com/alarm.asp (Last accessed 05/01/2012)

2. Maintaining Scotland's roads: a follow-up report, Audit Scotland, (2010) www.auditscotland.gov.uk/docs/central/2010/nr_110216_road_maintenance.pdf (Last accessed 05/01/2012)

3. J. Read and D. Whiteoak, The Shell Bitumen Handbook (fifth edition), Thomas Telford Publishing, London, (2003).

4. B. E. Ruth, Evaluation and Prevention of Water Damage to Asphalt Pavement Materials, American Society for Testing and Materials (ASTM) special technical publication 899, (1985).

5. T. W. Kennedy, F. L. Roberts and K. W. Lee, Evaluation of Moisture Effects on Asphalt Concrete Mixtures, Transportation Research Record, 911, 134-143 (1983).

6. G. D. Airey, A. C. Collop, S. E. Zoorob and R. C. Elliott, The Influence of Aggregate, Filler and Bitumen on Asphalt Mixture Moisture Damage, Construction and Building Materials, 22, 2015-2024, (2008).

7. S. Abo-Qudais, H. Al-Shweily, Effect of Aggregate Properties on Asphalt Mixtures Stripping and Creep Behaviour, Construction and Building Materials, 21, 1886-1898, (2007).

8. M. Horgnies, E. Darque-Ceretti, H. Fezai and E. Felder, Influence of the Interfacial Composition on the Adhesion Between Aggregates and Bitumen: Investigations by EDX, XPS and Peel Tests, International Journal of Adhesion \& Adhesives, 31, 238247, (2011).

9. J. C. Petersen, H. Plancher, E. K. Ensley, R. L. Venables and G. Miyake, Chemistry of Asphalt-Aggregate Interaction: Relationship with Pavement Moisture-Damage Prediction Test, Transportation Research Record, 843, 95-104,(1982).

10. S. Huang, R. E. Robertson, J. F. Branthaver and J. C. Petersen, Impact of Lime Modification of Asphalt and Freeze-Thaw Cycling on the Asphalt-Aggregate Interaction and Moisture Resistance to Moisture Damage, Journal of Materials in Civil Engineering, 17, 711-718, (2005).

11. D. W. Gilmore, J. B. Darland, Jr. L. M. Girdler, D. W. Wilson and J. A. Scherocaman, Changes in Asphalt Concrete Durability Resulting from Exposure to Multiple Cycles of Freezing and Thawing, ASTM special technical publication 899, 73-88, (1985).

12. G. D. Airey and Y. Choi, State of the Art Report on Moisture Sensitivity Test Methods for Bituminous Pavement Materials, International Journal of Road Materials and Pavement Design, 3, 355-72, (2002).

13. T. W. Kennedy, F. L. Roberts and K. W. Lee, Evaluating Moisture Susceptibility of Asphalt Mixtures Using the Texas Boiling Test, Transportation Research Record, 968, 45-54, (1984).

14. M. Solaimanian, J. Harvey, M. Tahmoressi, and V. Tandon, Test Methods to Predict Moisture Sensitivity of Hot Mix Asphalt Pavements. Proceedings of National Seminar on Moisture Sensitivity of Asphalt Pavements, 77-110, California, (2003).

15. T. Aschenbrener, Evaluation of Hamburg Wheel-Tracking Device to Predict Moisturedamage in Hot-Mix Asphalt, Transportation Research Record, 1492, 193-201,(1995).

16. A. C. Collop, Y. Choi, G. D. Airey and R. C. Elliott, Development of the Saturation Ageing Tensile Stiffness (SATS) Test, ICE Journal of Transport, 157, 163-171, (2004).

17. G. D. Airey, Y. Choi, A. C. Collop, A. J. V. Moore and R. C. Elliott, Combined Laboratory Ageing / Moisture Sensitivity Assessment of High Modulus Base Asphalt Mixtures, Journal of the Association of Asphalt Paving Technologists, 74, 307-345, (2005). 
18. American Association of State Highways and Transportation Officials, Resistance of Compacted Bituminous Mixture to Moisture Induced Damage, AASHTO T283-99, USA, (2000).

19. S. Caro, E. Masad, A. Bhasin and D. N. Little, Moisture Susceptibility of Asphalt Mixtures, Part 1: Mechanisms, International Journal of Pavement Engineering, 9, 8198, (2008).

20. S. Caro, E. Masad, A. Bhasin and D.N. Little, Moisture Susceptibility of Asphalt Mixtures, Part 2: Characterisation and Modelling, International Journal of Pavement Engineering, 9, 99-114, (2008).

21. S. Caro, E. Masad, A. H. Bhasin and D. Little, Micromechanical Modelling of the Influence of Material Properties on Moisture-Induced Damage in Asphalt Mixtures, Construction and Building Materials, 24, 1174-1192, (2010).

22. E. Masad and A. Al-Omari and H. C. Chen, Computations of Permeability Tensor Coefficients and Anisotropy of Hot Mix Asphalt Based on Microstructure Simulation of Fluid Flow, Computational Materials Science, 40, 449-459, (2007).

23. M. E. Kutay, A. H. Aydilek and E. Masad, Computational and Experimental Evaluation of Hydraulic Conductivity Anisotropy in Hot-mix Asphalt, International journal of Pavement Engineering, 8, 29-43, (2007).

24. T. W. Kennedy, F. L. Roberts, and K. W. Lee, Evaluation of Moisture Susceptibility of Asphalt Mixtures Using the Texas Freeze-Thaw Pedestal Test, Proceedings of the Association of Asphalt Paving Technologists, 51, 327-341, (1982).

25. D. G. Tunnicliff, and R. E. Root, Anti-Stripping Additives in Asphalt Concrete-State of Art 1981, Proceedings of the Association of Asphalt Paving Technologists, 51, 265293, (1982).

26. J. H. Fromm, The Mechanisms of Asphalt Stripping From Aggregate Surfaces, Proceedings of the Association of Asphalt Paving Technologists, 43, 191-196, (1974).

27. K. Kanitpong, and H. U. Bahia, Role of Adhesion and Thin Film Tackiness of Asphalt Binders in Moisture Damage of HMA, Proceedings of the Association of Asphalt Paving Technologists, 72, 502-528, (2003).

28. BS 7991, Determination of the mode I adhesive fracture energy, $\mathrm{G}_{\mathrm{IC}}$, of structural adhesives using the double cantilever beam (DCB) and tapered double cantilever beam (TDCB) specimens, BSI: London (2001).

29. BS EN 28510-1, Adhesives - Peel test for a flexible-bonded-to-rigid test specimen assembly - Part I: $90^{\circ}$ peel, BSI: London (1993).

30. ISO 8510-1, Adhesives - Peel test for a flexible-bonded-to-rigid test specimen assembly - Part I: $90^{\circ}$ peel, ISO: Geneva (1990).

31. D. R. Moore and J. G. Williams, A Protocol for Determination of the Adhesive Fracture Toughness by Peel Testing of Flexible Laminates, Imperial College London, (2007). http://www3.imperial.ac.uk/meadhesion/testprotocols/peel (Last accessed 13/10/2011)

32. A. J. Kinloch, C. C. Lau and J. G. Williams, The Peeling of Flexible Laminates, International Journal of Fracture, 66, 45-70, (1994).

33. IC Peel software, http://www3.imperial.ac.uk/meadhesion/testprotocols/peel (Last accessed 13/10/2011).

34. B. Sengoz and E. Agar, Effect of Asphalt Film Thickness on the Moisture Sensitivity Characteristics of Hot-Mix Asphalt, Building and Environment, 42, 3621-3628, (2007).

35. T. Igarashi, Mechanics of peeling of rubbery materials. I. Peel strength and energy dissipation, Journal of Polymer Science: Polymer Physics Edition 13, 2129-2134, (1975).

36. S. Giannis, R. D. Adams, L. J. Clark, and M.A. Taylor, Peel Behaviour of Aircraft Fuel Tank Sealants: the Effect of Peel Angle, Sealant Layer Thickness and Peel Rate, Journal of Adhesion Science and Technology 22, 1495-1522, (2008). 


\section{Figures}

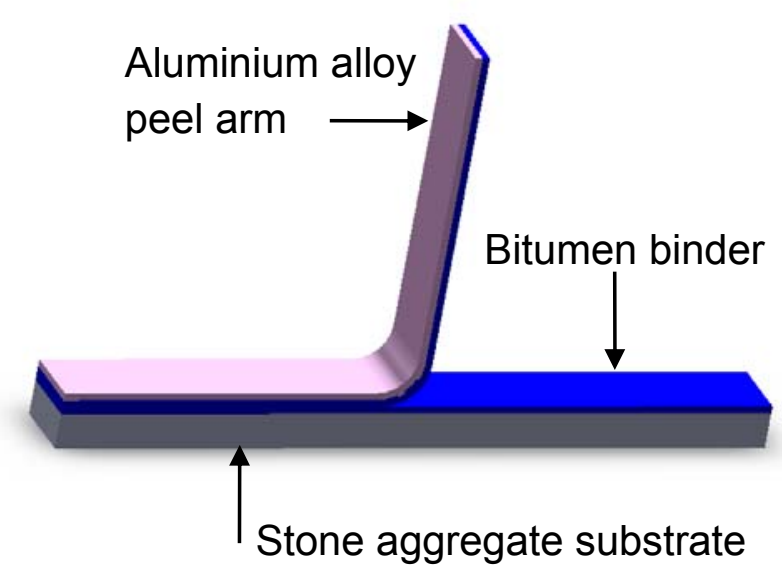

Figure 1. Schematic diagram of a $90^{\circ}$ peel test specimen. 
(a)

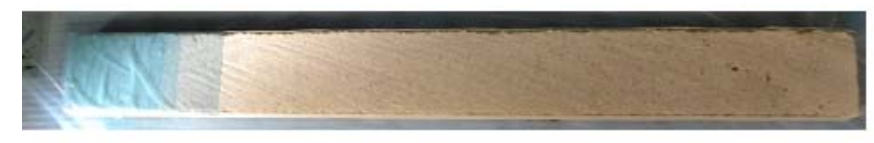

(b)

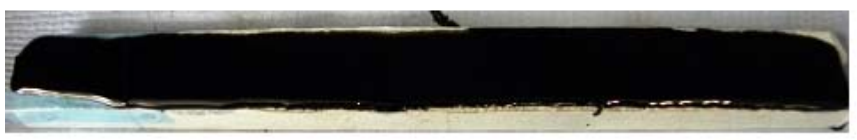

(c)

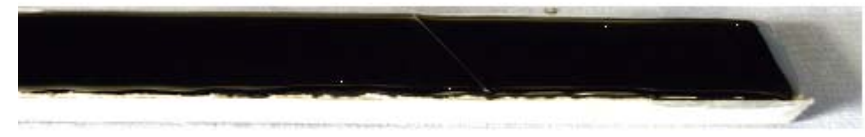

(d)

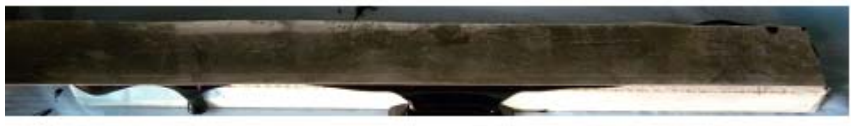

(e)

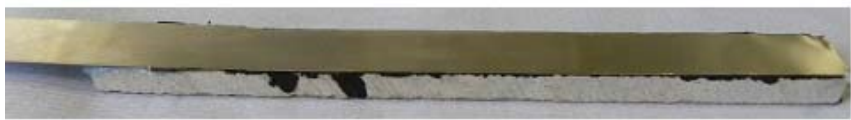

Figure 2. Stages in the manufacture of the peel specimen, (a) preparation of the aggregate, (b) applying the pre-heated bitumen onto the aggregate, (c) placing the wire spacers, (d) placing the peel arm on the top and (e) a ready-to-test specimen. 
(a)

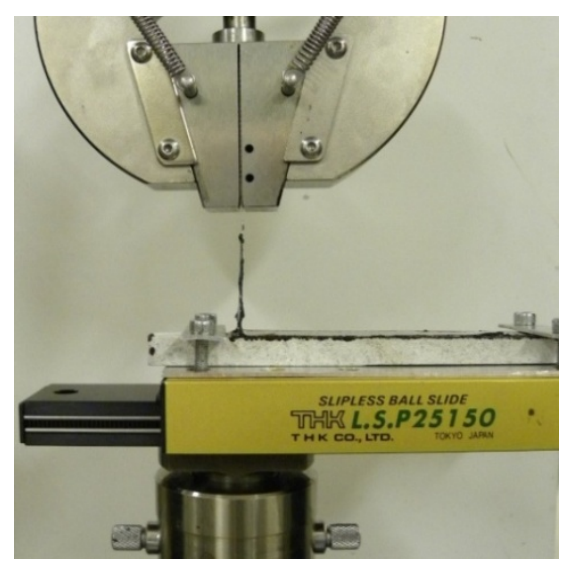

(b)

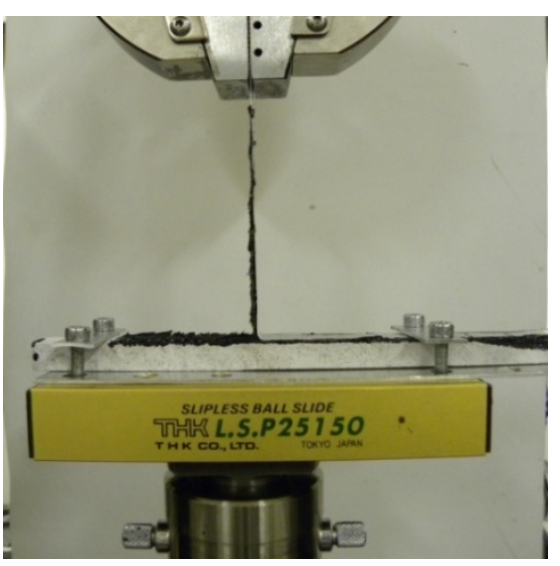

Figure $3.90^{\circ}$ peel testing, (a) before test and (b) during test. 


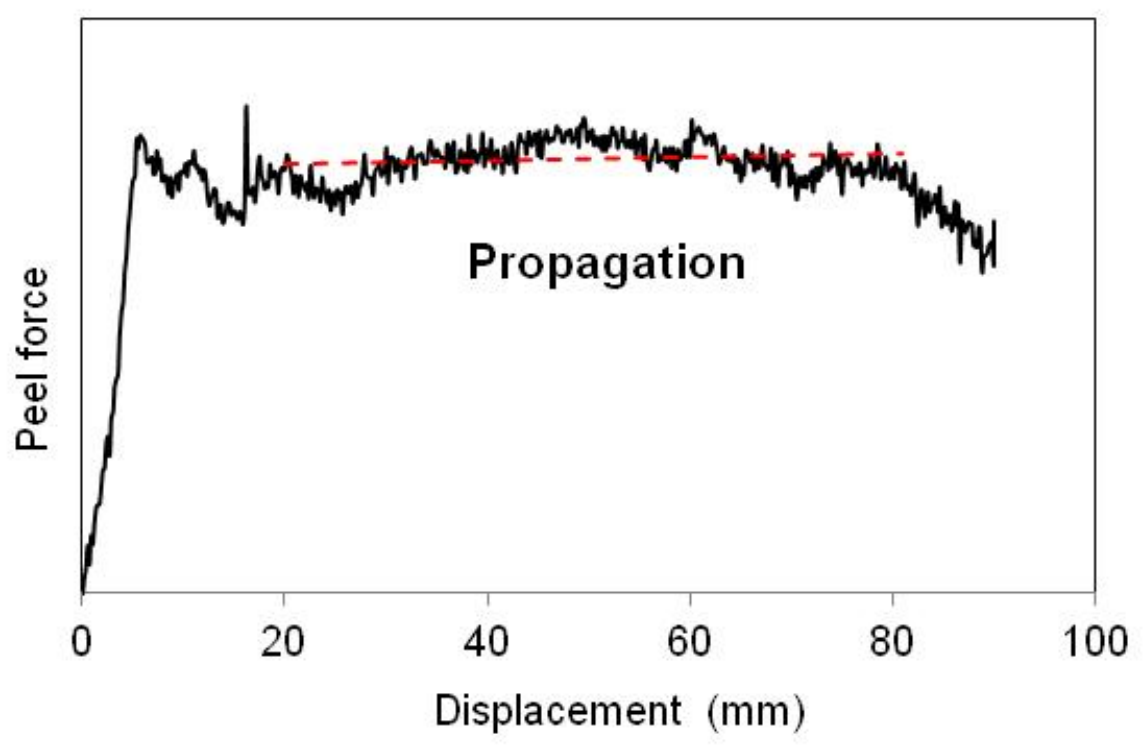

Figure 4. Peel force versus displacement in a peel test. 
(a)

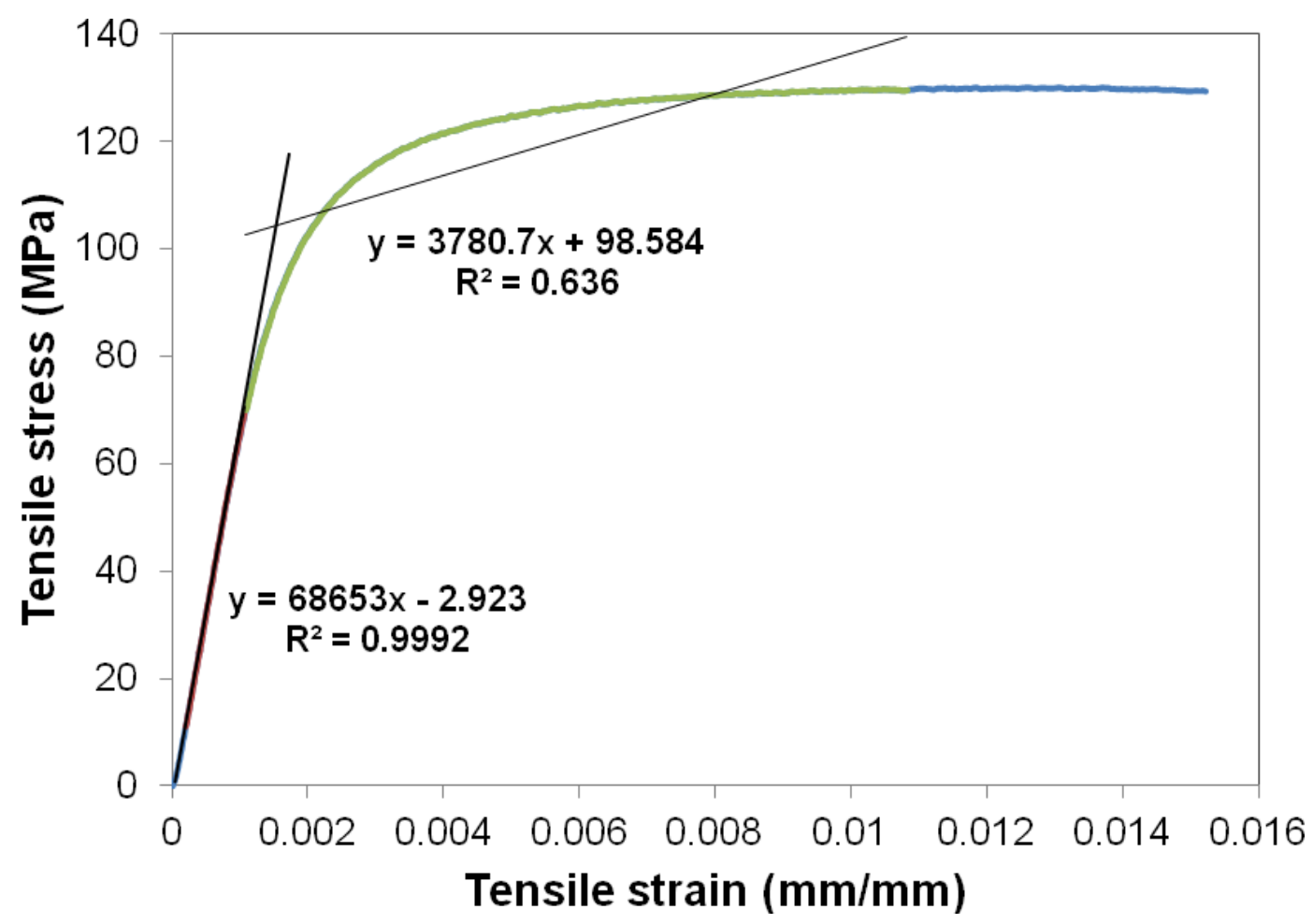

(b)

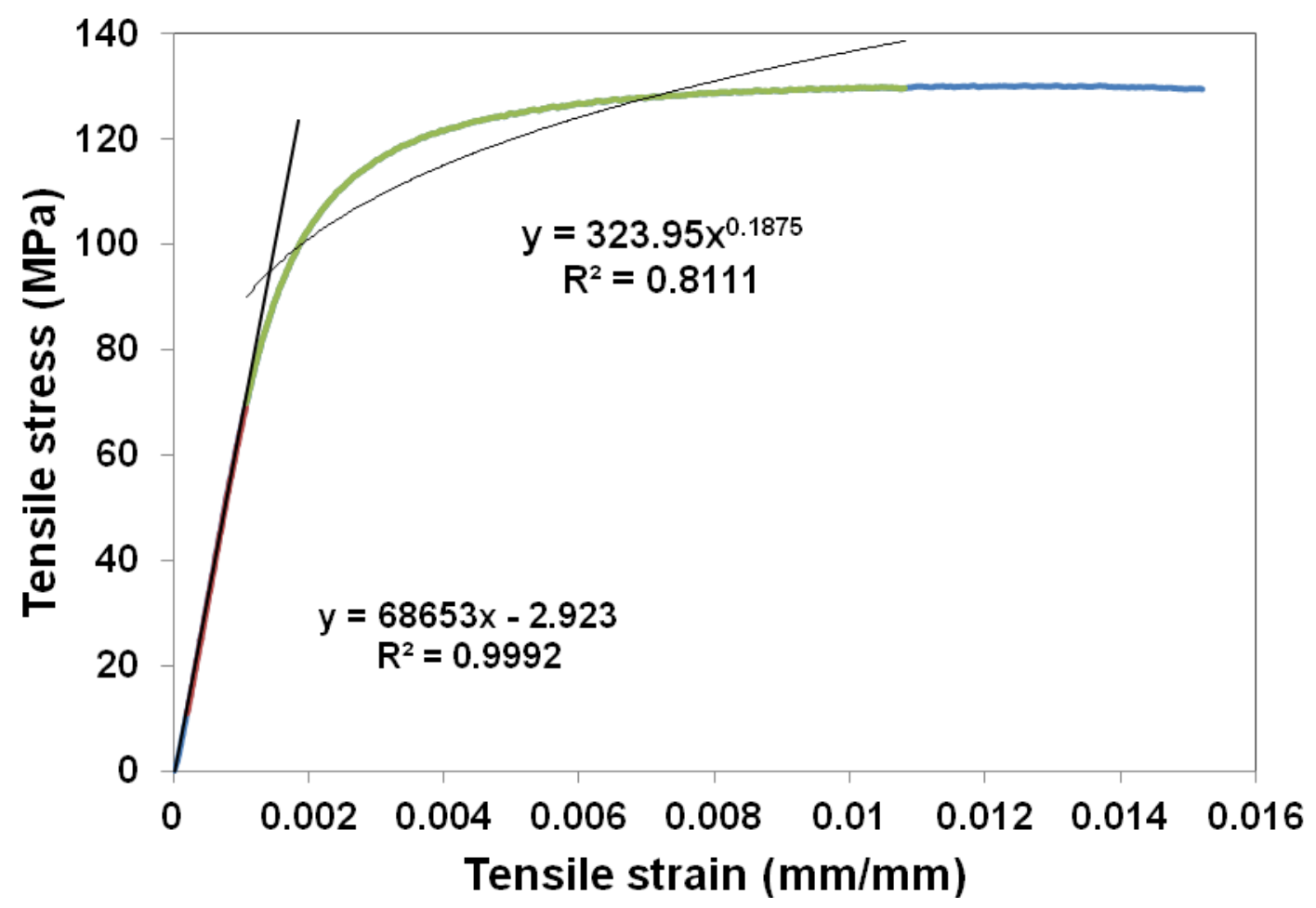

Figure 5. Measured tensile stress-strain curve for the aluminium peel arm: (a) fitted using the bi-linear model and (b) fitted using the power-law model. 
(a)

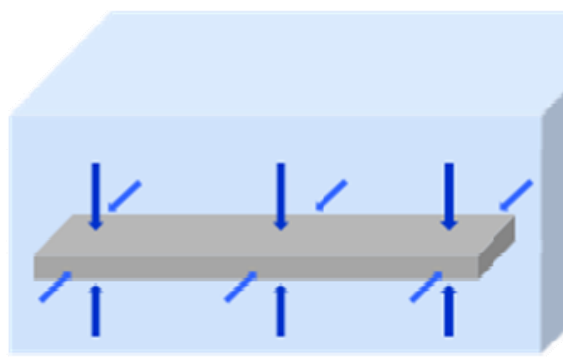

Aggregate submersed in a water bath

(b)

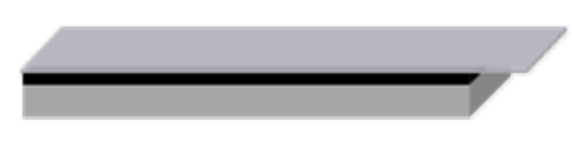

Bonded joint

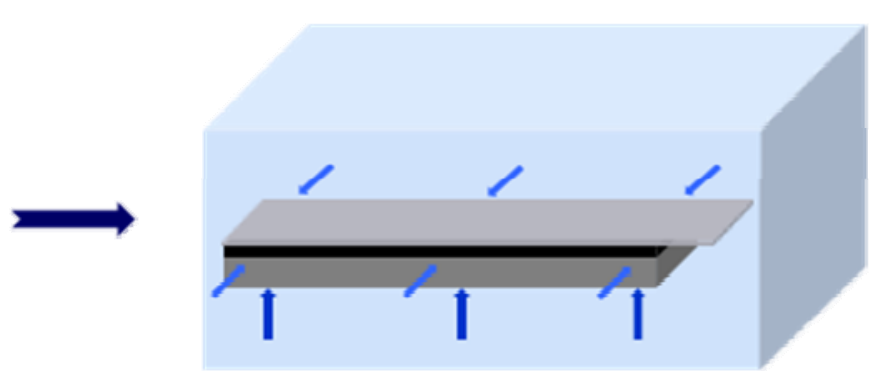

Submersed in a water bath

Figure 6. Schematic diagrams of (a) pre-bond moisture conditioning: immersing the aggregate only prior to bonding, and (b) post-bond moisture conditioning: immersing the completed bitumen-aggregate joint. 
(a)

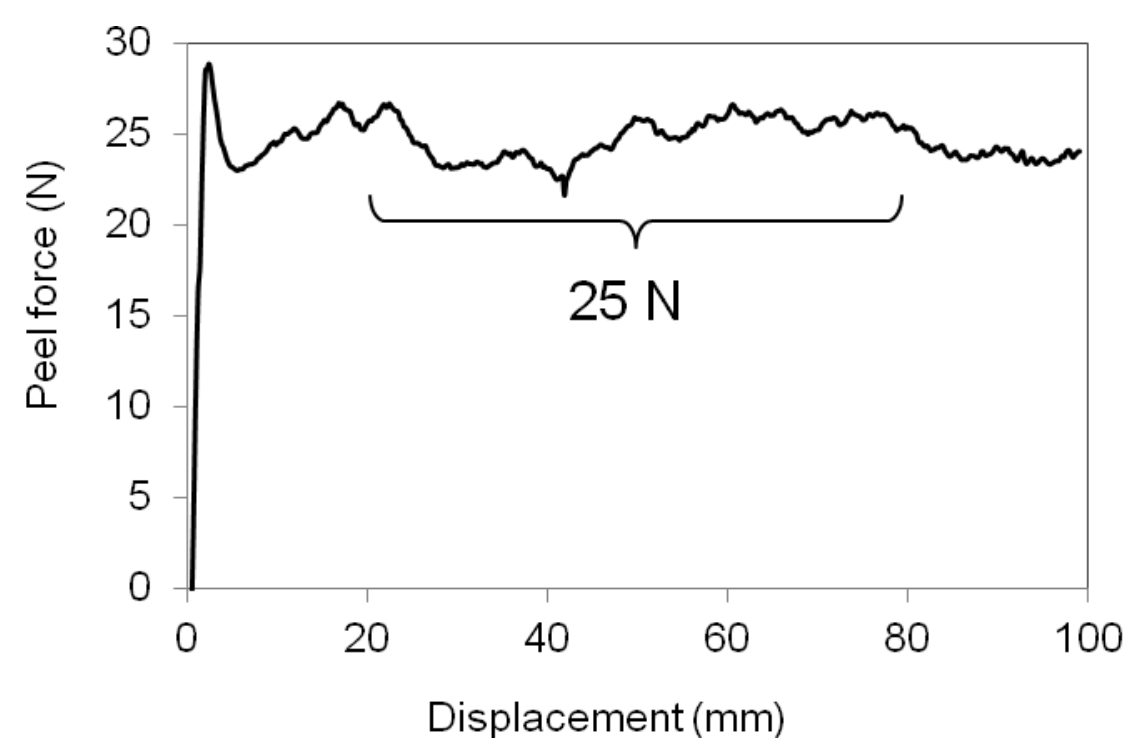

(b)

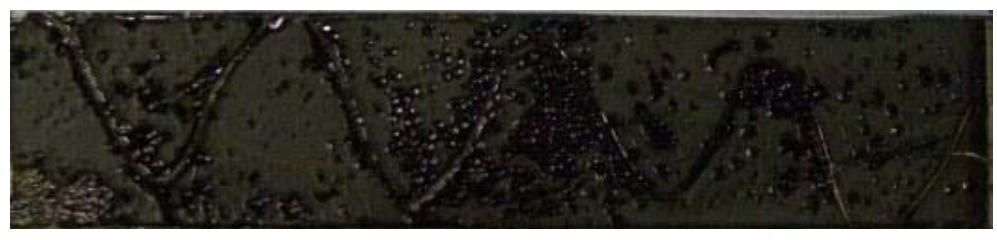

(c)

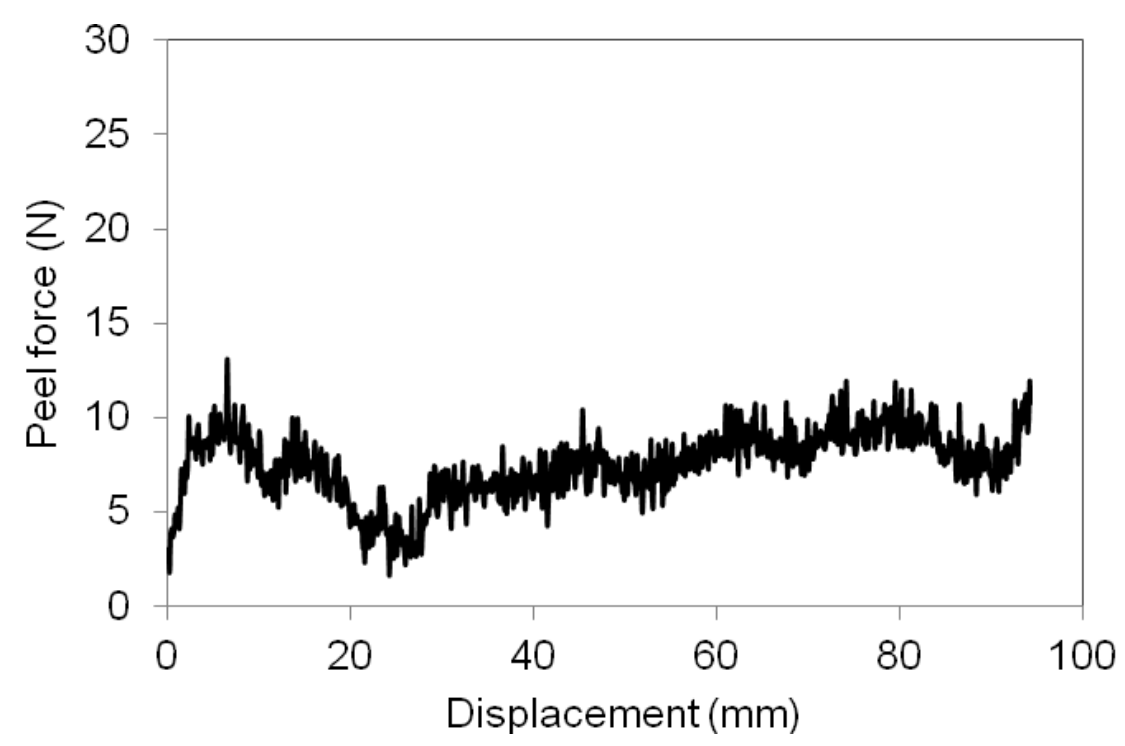

(d)

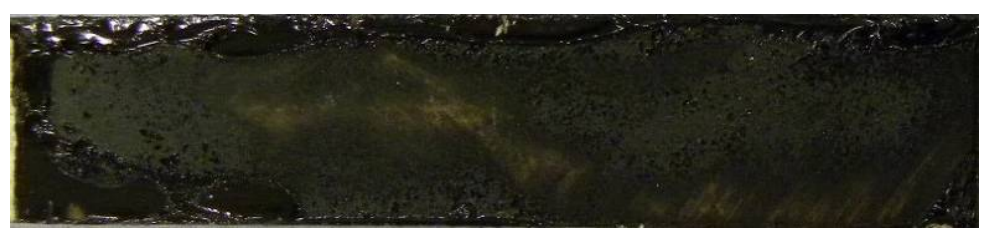

Figure 7. Examples of peel results: $(a, b)$ a satisfactory peel curve and the corresponding aggregate fracture surface; and (c, d) an unsatisfactory peel curve and the corresponding aggregate fracture surface. 
(a)

(b)

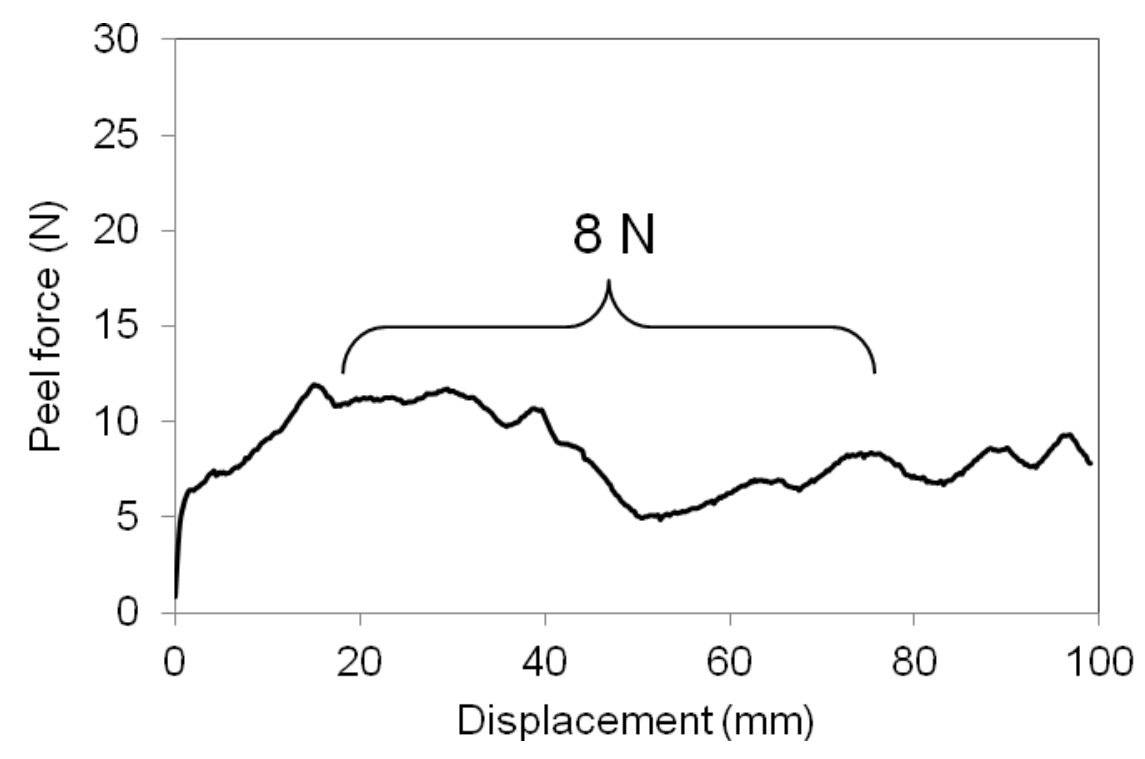

(c)
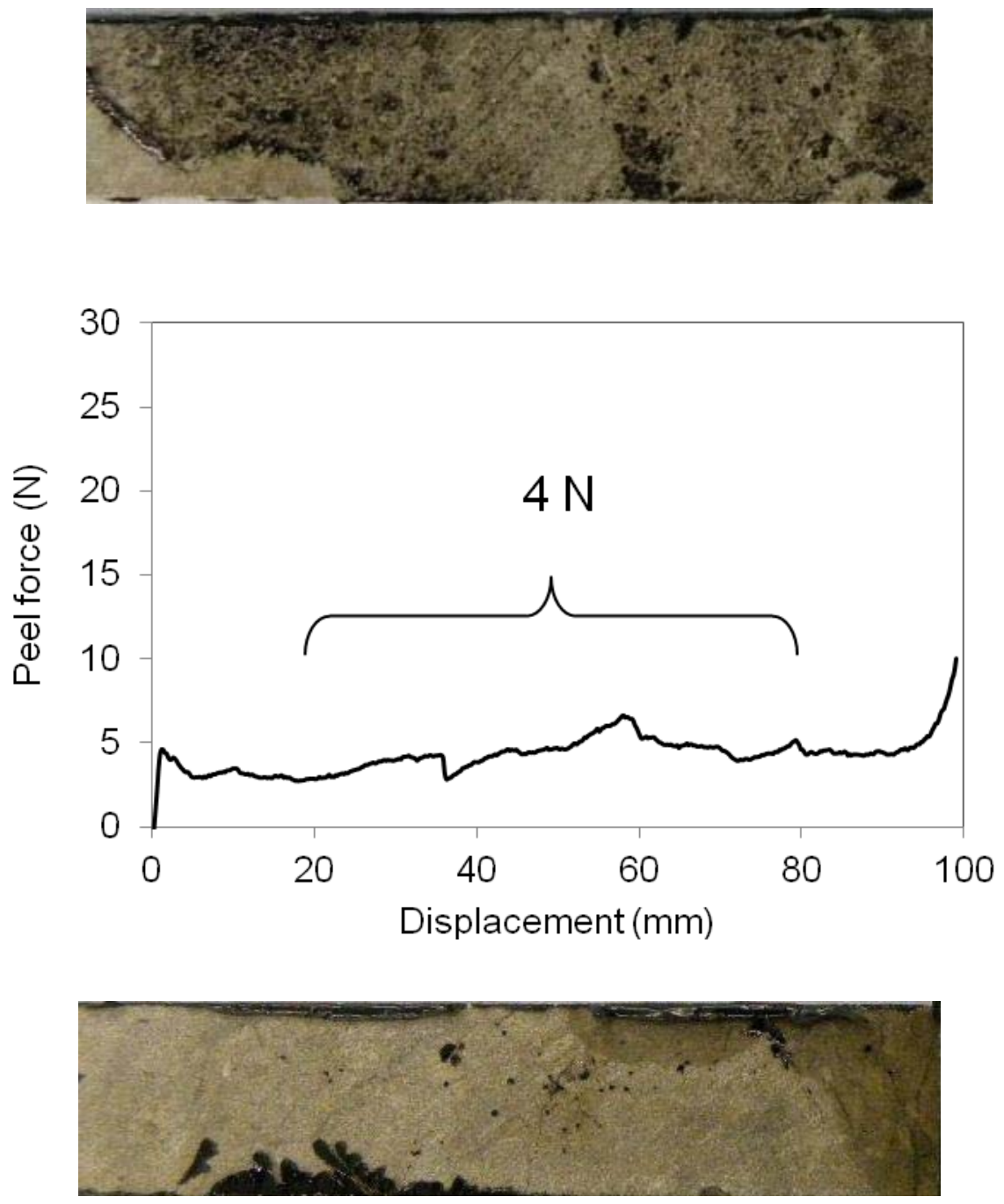

Figure 8. Peel curves and images of fracture surfaces of a 40/60 pen bitumen-limestone specimen tested at a speed of $10 \mathrm{~mm} / \mathrm{min}$ : $(a, b)$ pre-bond moisture conditioning for 7 days; (c, d) post-bond moisture conditioning for 7 days. 
(a)

(b)

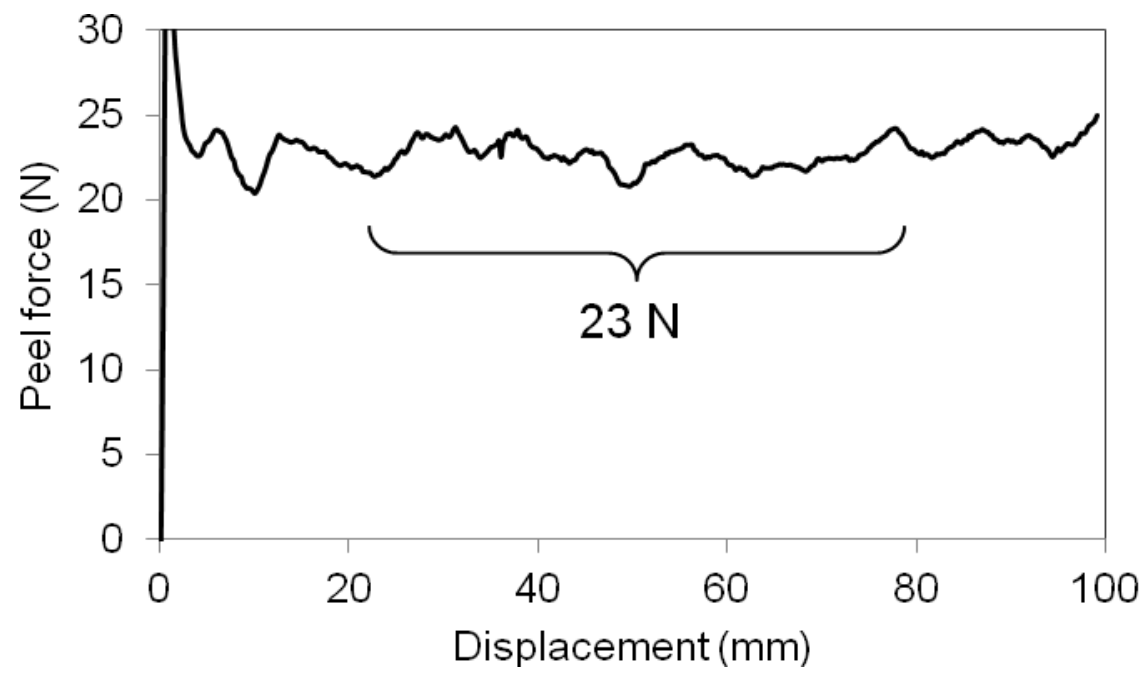

Figure 9. Peel results of a 40/60 pen bitumen-aluminium substrate specimen tested at a speed of $10 \mathrm{~mm} / \mathrm{min}$, post-bond moisture conditioning for 7 days, (a) peel curve and (b) the corresponding fracture surface of the aluminium substrate. 
(a)

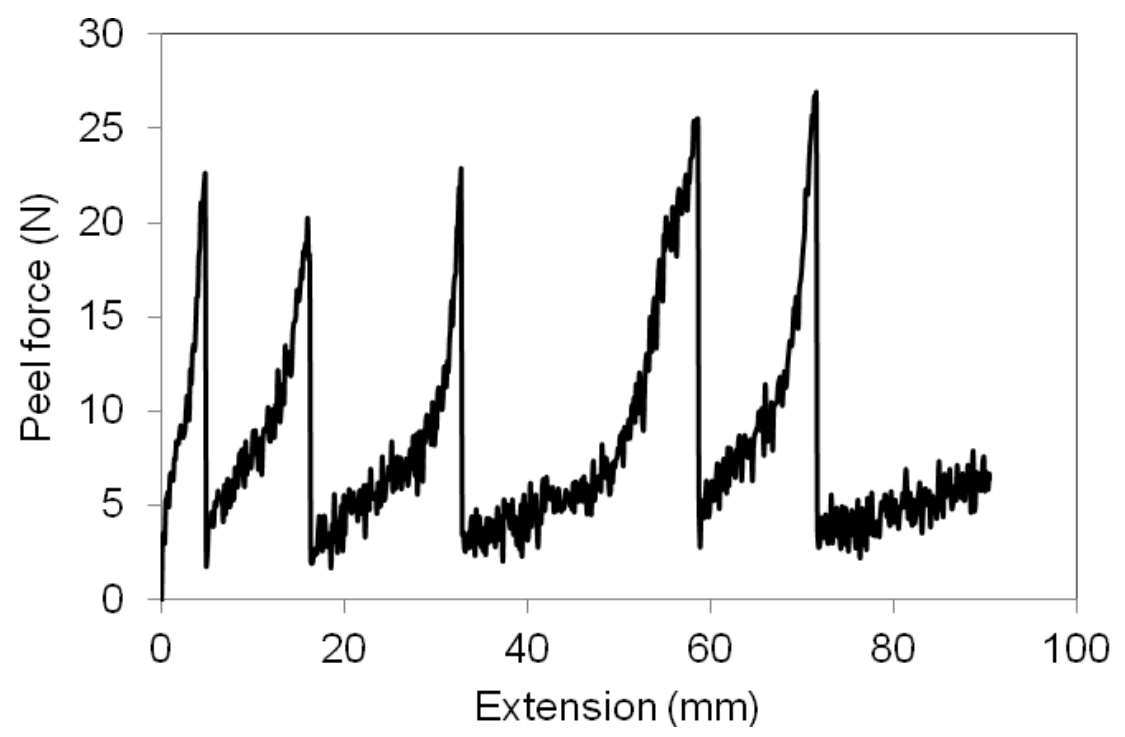

(b)

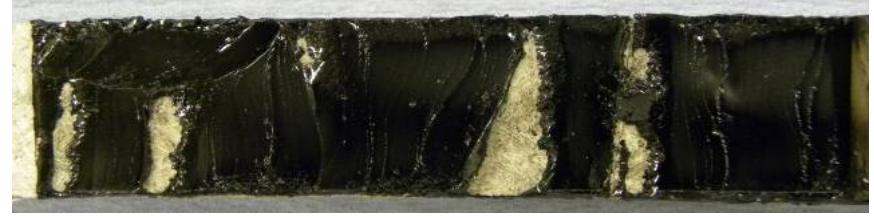

(c)

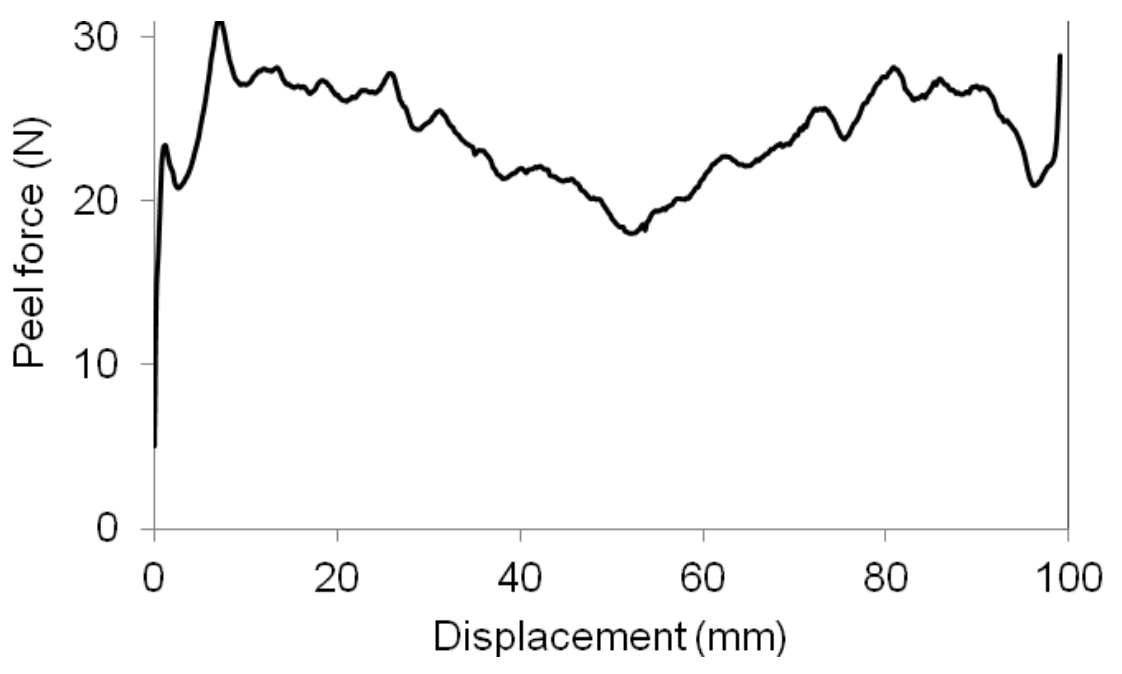

(d)

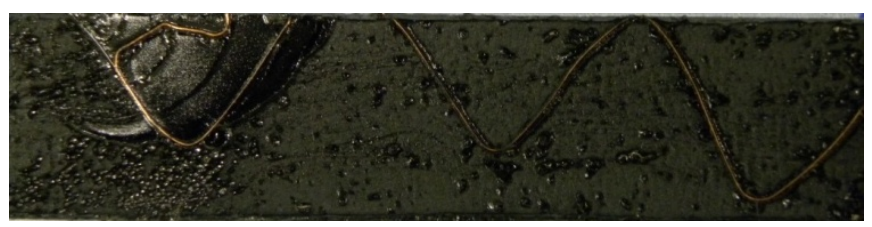

Figure 10. Peel curves and images of fracture surfaces of a 10/20 pen bitumen-limestone specimen; $(a, b)$ tested at a speed of $10 \mathrm{~mm} / \mathrm{min}$, and (c, d) tested at a speed of $2 \mathrm{~mm} / \mathrm{min}$. 


\section{Tables}

Table 1. The fitting of the stress-strain curve for the aluminium peel arm, and the resulting values of $G_{p}$ and $G_{A}$ for the same test specimen on a joint using $40 / 60$ pen bitumen.

\begin{tabular}{|c|c|c|}
\hline & Bi-linear & Power Law \\
\hline$E_{1}(\mathrm{GPa})$ & 69 & 69 \\
\hline$\alpha$ & 0.055 & - \\
\hline$n$ & - & 0.1875 \\
\hline$G\left(\mathrm{~J} / \mathrm{m}^{2}\right)$ & 1150 & 1150 \\
\hline$G_{\mathrm{p}}\left(\mathrm{J} / \mathrm{m}^{2}\right)$ & 505 & 517 \\
\hline$G_{\mathrm{A}}\left(\mathrm{J} / \mathrm{m}^{2}\right)$ & 645 & 633 \\
\hline
\end{tabular}

Table 2. Peel results of dry and moisture-conditioned peel joints made of $40 / 60$ pen bitumen binder bonded to the limestone aggregate substrate, tested at $10 \mathrm{~mm} / \mathrm{min}$.

\begin{tabular}{|l|c|c|c|c|c|l|}
\hline Conditioning & Time & $P$ & \multicolumn{2}{|c|}{$G_{\mathrm{A}}\left(\mathrm{J} / \mathrm{m}^{2}\right)$} & $G_{\mathrm{A}} / G_{\mathrm{A} 0}$ & Locus of failure from direct \\
& (days) & $(\mathrm{N})$ & Mean & $\mathrm{SD}$ & & observation \\
\hline Dry & 0 & 23 & 633 & 34 & 1.00 & Cohesive \\
\hline Pre-bond conditioned & 3 & 5 & 89 & 20 & 0.14 & Mainly interfacial failure \\
\cline { 2 - 8 } & 7 & 6 & 111 & 69 & 0.18 & Interfacial failure \\
\hline Post-bond conditioned & 1 & 10 & 212 & 50 & 0.33 & Mainly interfacial, some cohesive \\
\cline { 2 - 8 } & 3 & 11 & 240 & 50 & 0.38 & Mainly interfacial \\
\cline { 2 - 7 } & 5 & 8 & 160 & 34 & 0.25 & Interfacial failure \\
\cline { 2 - 7 } & 7 & 8 & 160 & 90 & 0.25 & Interfacial failure \\
\cline { 2 - 7 } & 10 & 4 & 69 & 20 & 0.11 & Interfacial failure \\
\hline
\end{tabular}


Table 3. Peel results of dry and moisture-conditioned peel joints made of $40 / 60$ pen binder bonded to the aluminium substrate, tested at $10 \mathrm{~mm} / \mathrm{min}$.

\begin{tabular}{|l|c|c|c|c|c|l|}
\hline Conditioning & Time & $P$ & \multicolumn{2}{|c|}{$G_{\mathrm{A}}\left(\mathrm{J} / \mathrm{m}^{2}\right)$} & $G_{\mathrm{A}} / G_{\mathrm{A} 0}$ & Locus of failure from direct \\
& (days) & $(\mathrm{N})$ & Mean & $\mathrm{SD}$ & & observation \\
\hline Dry & 0 & 23 & 633 & 20 & 1.00 & Cohesive \\
\hline Post-bond conditioned & 1 & 23 & 633 & 69 & 1.00 & Cohesive \\
\cline { 2 - 7 } & 3 & 21 & 562 & 20 & 0.89 & Cohesive \\
\cline { 2 - 7 } & 5 & 22 & 597 & 20 & 0.94 & Cohesive \\
\cline { 2 - 7 } & 7 & 22 & 597 & 0 & 0.94 & Cohesive \\
\cline { 2 - 7 } & 10 & 23 & 633 & 20 & 1.00 & Cohesive \\
\hline
\end{tabular}

Table 4. Peel results of bitumen binders of different penetration grades bonded to the limestone aggregate substrate, tested at 2 or $10 \mathrm{~mm} / \mathrm{min}$ as shown.

\begin{tabular}{|l|c|c|c|l|}
\hline Binder & $P$ & \multicolumn{2}{|c|}{$G_{\mathrm{A}}\left(\mathrm{J} / \mathrm{m}^{2}\right)$} & Locus of failure from direct \\
& $(\mathrm{N})$ & Mean & $\mathrm{SD}$ & observation \\
\hline 10/20 pen (Hard, $R=2 \mathrm{~mm} / \mathrm{min})$ & 25 & 705 & 69 & Cohesive failure \\
\hline 40/60 pen (Medium, $R=10 \mathrm{~mm} / \mathrm{min})$ & 23 & 633 & 34 & Cohesive failure \\
\hline $70 / 100$ pen (Soft, $R=10 \mathrm{~mm} / \mathrm{min})$ & 20 & 527 & 34 & Cohesive failure \\
\hline
\end{tabular}


Appendix 1: Test report for typical dry peel test specimen, 40/60 pen bitumen-limestone joint.

Data from the peel test

\begin{tabular}{|c|c|c|c|}
\hline \multicolumn{2}{|l|}{ Parameter } & Value & Units \\
\hline \multicolumn{2}{|l|}{ Peel angle } & 90 & $\circ$ \\
\hline \multicolumn{2}{|l|}{ Test speed, $R$} & 10 & $\mathrm{~mm} / \mathrm{min}$ \\
\hline \multicolumn{2}{|l|}{ Test temperature } & 20 & ${ }^{\circ} \mathrm{C}$ \\
\hline \multicolumn{2}{|l|}{ Specimen length, $L$} & 200 & $\mathrm{~mm}$ \\
\hline \multicolumn{2}{|l|}{ Specimen width, $b$} & 20.0 & $\mathrm{~mm}$ \\
\hline \multicolumn{2}{|c|}{ Aluminium peel arm thickness, $h$} & 0.20 & $\mathrm{~mm}$ \\
\hline \multicolumn{2}{|c|}{ Thickness of adhesive layer, $h_{\mathrm{A}}$} & 0.25 & $\mathrm{~mm}$ \\
\hline \multicolumn{2}{|c|}{ Modulus of the adhesive, $E_{\mathrm{A}}$} & 1.5 & $\mathrm{MPa}$ \\
\hline \multicolumn{2}{|c|}{ Peel force, $P$} & 23 & $\mathrm{~N}$ \\
\hline \multirow{3}{*}{$\begin{array}{l}\text { Tensile properties of the } \\
\text { peel arm }\end{array}$} & Elastic modulus, $E_{1}$ & 69 & $\mathrm{GPa}$ \\
\hline & $\mathrm{n}$ & 0.1875 & \\
\hline & Yield stress, $\sigma_{y}$ & 95 & $\mathrm{GPa}$ \\
\hline
\end{tabular}

Derived results by calculations (for each of the three fits to the stress-strain data)

\begin{tabular}{|l|c|l|}
\hline Parameter & Value & Units \\
\hline Input energy, $G$ & 1150 & $\mathrm{~J} / \mathrm{m}^{2}$ \\
\hline Plastic work, $G_{\mathrm{p}}$ & 517 & $\mathrm{~J} / \mathrm{m}^{2}$ \\
\hline Adhesive fracture energy, $G_{\mathrm{A}}$ & 633 & $\mathrm{~J} / \mathrm{m}^{2}$ \\
\hline Correction factor, $G_{\mathrm{p}} / G$ & 45 & $\%$ \\
\hline
\end{tabular}

Notes:

1. The elastic modulus of the adhesive, $E_{\mathrm{A}}$, was $1.5 \mathrm{MPa}$ for the $40 / 60$ pen bitumen at a tensile strain rate of $10 \mathrm{~mm} / \mathrm{min}$ and a temperature of $20^{\circ} \mathrm{C}$, which was determined from the tensile tests conducted using the $40 / 60$ pen bitumen binder.

2. The adhesive fracture energy was determined according to Equation 2. Ideally, the corrections for plastic deformation should not be too large otherwise errors for the determination of adhesive fracture energy will become significant. The size of this correction is given by $\left(G_{p} / G\right) \times 100 \%$; the smaller this correction the better [31]. 